\title{
Nachwuchspreis der DeGEval 2019
}

\author{
Edith Halves
}

Beim Nachwuchspreis in diesem Jahr sind Kontinuität und Wandel sehr eng miteinander verzahnt. Kontinuität, weil der mittlerweile 13. Preis auf der 22. Jahrestagung vergeben wird. Wandel, weil drei Mitglieder der letztjährigen Jury kurzfristig aus persönlichen bzw. beruflichen Gründen nicht mehr zur Verfügung standen. Dankenswerterweise haben Herr Prof. Widmer und Frau Dr. Wroblewski sich sehr kurzfristig zur Mitarbeit bereit erklärt. Frau Prigge, Frau Halves, Mitglieder der Jury 2018, und Frau Flatters als Gewinnerin des Vorjahres haben die Jury vervollständigt.

Ziel des Preises ist es, jährlich eine Nachwuchsevaluatorin/einen Nachwuchsevaluator oder auch eine Nachwuchsgruppe auszuzeichnen und damit eine herausragende Arbeit im Bereich Evaluation im deutschsprachigen Raum zu würdigen. Aus Sicht der DeGEval soll dieser Preis zum einen die Bedeutung des wissenschaftlichen Nachwuchses auf dem Gebiet der Evaluation und zum anderen die Bedeutung der Nachwuchsförderung insgesamt hervorheben.

Für den Nachwuchspreis 2019 wurden insgesamt drei Beiträge eingereicht: je eine Dissertation, eine Masterarbeit und eine Praxisarbeit. Neben den unterschiedlichen Formaten wurden unterschiedliche Themenfelder behandelt. Dies stellte die Jury bei ihrer Entscheidung vor einige Herausforderungen. Schließlich hat sich die Jury für die Masterarbeit von Patricia Berndt entschieden.

Beim Wettbewerbsbeitrag handelt es sich um eine Abschlussarbeit im Rahmen des Masterstudiengangs Evaluation der Universität des Saarlandes und der Hochschule für Technik und Wirtschaft des Saarlandes. Betreut wurde die Arbeit von Prof. Filsinger. Gegenstand der Arbeit ist die Evaluation von Verstetigungsprozessen im Stadtbauförderungsprogramm „Soziale Stadt“ am Beispiel des Brandenburgischen Viertels in Eberswalde. Die Arbeit entstand im Kontext eines Auftrags der Stadt Eberswalde an „S.T.E.R.N. Gesellschaft der behutsamen Stadterneuerung $\mathrm{mbH}$ Berlin“, bei der die Wettbewerbsteilnehmerin beschäftigt ist.

Die Masterarbeit hat eine stark disziplinäre Orientierung am wissenschaftlichen Diskurs der Evaluation. Sowohl ausgewählte deutsche als auch internationale Literatur des Feldes werden genutzt, um das eigene Vorgehen, die Evaluation von Verstetigungsprozessen in einem Städtebauförderungsprogramm systematisch $\mathrm{zu}$ reflektieren. Vor dem Hintergrund einer multizentrischen Programmanlage und eines entsprechend komplexen Evaluationsdesigns können breit gefächerte Ergebnisse 
präsentiert werden. Durch die theoretischen Reflexionen werden die verschiedenen Vorgehensweisen in ihren Möglichkeiten und Grenzen im Feld der Städtebauförderung und der multizentrischen Programme eingeordnet. Diese Erkenntnisse, die aus der Verzahnung von Theorie und Praxis gewonnen wurden, stellen einen wichtigen Beitrag zur Entwicklung der Evaluation als Profession dar.

Die Arbeit liefert eine fundierte und sorgfältige Auseinandersetzung mit relevanten Beiträgen aus der Evaluationsliteratur und verortet die eigene Vorgehensweise in souveräner Weise. Die Autorin leistet eine kenntnisreiche Beschreibung des Evaluationsgegenstandes des Programms „Soziale Stadt“ und der Realisierung im Brandenburgischen Viertel. Sie diskutiert dabei das Evaluierungssystem der Städtebauförderung und entwickelt ein Wirkungsmodell als konzeptionelle Grundlage der eigenen Evaluation. Die Arbeit setzt sich darüber hinaus vertieft mit dem vielfältigen Konzept der ,Verstetigung ' auseinander. Neben diesen theoretisch-konzeptionellen Stärken überzeugt die Arbeit auch auf empirischer Ebene. Die Empirie umfasst Erhebungen mit qualitativen und quantitativen Instrumenten, deren Einsatz in gut nachvollziehbarer Weise beschrieben wird. Das so gewonnene Material erlaubt eine schlüssige Beantwortung der Untersuchungsfragen, die sich auf einer Metaebene befinden und sich mit den Möglichkeiten und Grenzen der Evaluation dieses Gegenstandes auseinandersetzen.

Bemerkenswert ist weiterhin, dass die Einzelarbeit von Frau Berndt ihren Stellenwert gegenüber und in Ergänzung zur Gesamtevaluation der S.T.E.R.N.-Gesellschaft ,behauptet".

Damit geht der diesjährige 13. DeGEval-Nachwuchspreis 2019 an Frau Patricia Berndt für ihre Masterarbeit mit dem Titel „Evaluation von Verstetigungsprozessen im Städtebauförderungsprogramm 'Soziale Stadt am Beispiel Brandenburgisches Viertel in Eberswalde“. Wir gratulieren Frau Berndt ganz herzlich zu dieser hervorragenden Forschungsarbeit und hoffen auf zahlreiche weitere Beiträge von ihr zur Praxis und Theorie der Evaluation.

Wir freuen uns, auch in diesem Jahr den Preis wieder an eine Masterarbeit vergeben zu können und hoffen damit auch weitere Studierende für eine Einreichung ihrer Masterarbeit für den nächstjährigen DeGEval-Nachwuchspreis zu motivieren.

Herzlichen Glückwunsch an Frau Berndt!

Die Jury des Nachwuchspreises der DeGEval e.V. 2019 